\title{
Two Cases of Cardiac Arteriovenous Malformation Complicated by a Local Angioproliferative Process
}

\author{
Lorine B. Meijer-Jorna • Renée B. A. van den Brink • \\ Anton E. Becker - Allard C. van der Wal
}

Received: 23 December 2009/Accepted: 27 March 2010/Published online: 16 April 2010

(C) The Author(s) 2010. This article is published with open access at Springerlink.com

\begin{abstract}
Vascular malformations of the heart are extremely rare with only a few cases of the arteriovenous type of vascular malformation (AVM) reported. We investigated the pathology of two additional cases, which were complicated by the occurrence of a local vasoproliferative response of immature but benign vessels. We suppose that the mass forming effect of this vasoproliferative response, which has also been reported recently as a complication of congenital AVM elsewhere in the body, has significantly contributed to the onset of symptoms and ultimate death of both patients.
\end{abstract}

Keywords Angiogenesis - Arteriovenous malformation · Congenital $\cdot$ Heart

Vascular malformations (VMs) are congenital anomalies composed of mature but often dysplastic blood and/or lymphatic vessels that result from localized errors of angiogenesis during embryonic development [12]. VMs may occur at any topographic site and are categorized according to the dominant types of vessels that make up the lesion (capillary, arteriovenous, venous, lymphatic, or mixed). Moreover, arteriovenous malformations (AVMs) may have aberrant direct connections (shunts) between veins and arteries without an intervening capillary bed.

L. B. Meijer-Jorna · A. E. Becker · A. C. van der Wal ( $\square)$ Department of Pathology, Academic Medical Center, University of Amsterdam, 1100 DD Amsterdam, The Netherlands

e-mail: a.c.vanderwal@amc.uva.nl

R. B. A. van den Brink

Department of Cardiology, Academic Medical Center,

University of Amsterdam, 1100 DD Amsterdam,

The Netherlands
True cardiac AVMs, which are intramural conglomerates of malformed arteries and veins fed by one of the coronary arteries, have only been reported incidentally $[1,4,8]$.

We retrieved two cases of cardiac AVM from the autopsy files of our hospital. They are of particular interest because there was development of an additional benign angioproliferative mass within the boundaries of the congenital lesion.

\section{Case Reports}

Case No. 1

A 22-year old man without medical history was admitted to the hospital with symptoms of cardiac failure that had developed during a period of weeks. Echocardiography showed pericardial effusions, and a tumorous process at the apex of the heart was suspected. Coronary angiography showed a hypervascular mass, which was limited to the perfusion territory of the right coronary artery.

Based on the suspicion of malignancy, endomyocardial biopsy specimens were taken and showed increased immature capillary vessels lined with plump anti-CD31 immunopositive endothelium amidst pre-existing hypertrophied myocardium. Proliferative activity of the vessels was visualized by nuclear protein $\mathrm{Ki}-67$ immunostaining in a small fraction of the endothelial cells. Anti-smooth muscle actin immunostaining showed evident pericytic lining of all microvessels, which is characteristic for a benign vascular process (Fig. 1a through c). A working diagnosis of a benign proliferative lesion (angiomatosis) was made.

A pericardial biopsy specimen taken 1 week later during a pericardial window procedure for pericardial effusion 
Fig. 1 Patient no. 1. a Endomyocardial biopsy specimen showing diffuse spread of capillary vessels amidst myocardium (haematoxylin and eosin stain). b Immunostain with anti-CD31 antibody outlines the endothelium of the immature vessels. c Immunostain with anti-Ki-67 shows nuclear positivity in some vascular wall cells. d Midventricular transverse section through the heart showing biventricular endocardial fibroelastosis and cystically dilated vessels. e Elastica von Gieson-stained section of myocardium showing closely apposed artery and vein (with recanalized thrombus [arrow]) of the vascular malformation
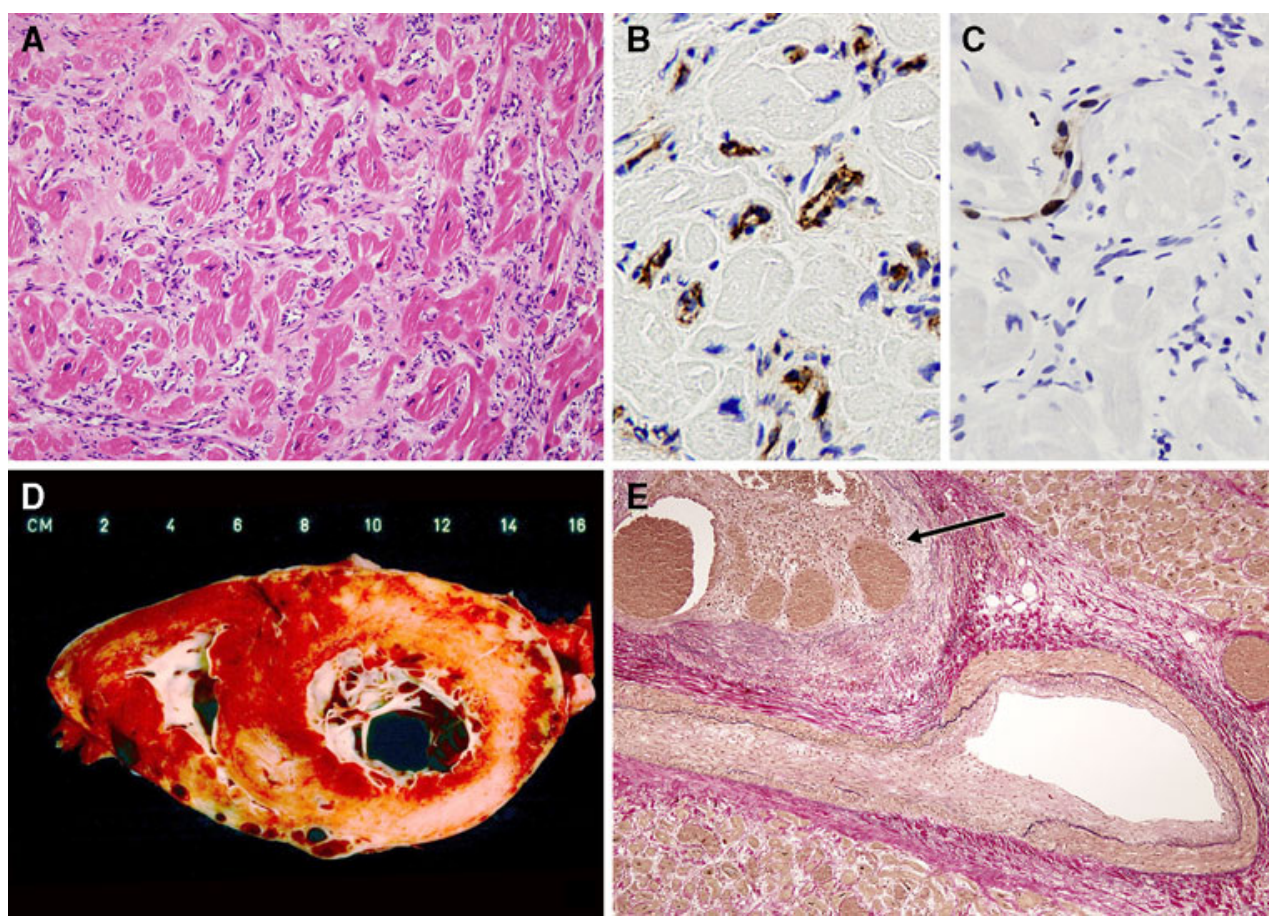

(600 cc daily) showed a convolute of thin-walled mature vessels that clearly differed from the immature type of microvessels noticed in the initial myocardial biopsy. Due to likely extension of the vascular process in the pericardium, the patient was rejected for heart transplantation. The patient's condition gradually worsened, and he eventually died from intractable pump failure.

Permission for autopsy of the heart only was obtained postmortem. The heart showed immense hypertrophy (weight $1,360 \mathrm{~g}$ ), dilatation of both ventricles, and firm fibrous pericardial adhesions, mainly at the apex. Transverse slicing of both ventricles showed asymmetric thickening of the wall, cystically dilated intramural blood vessels, and prominent endocardial fibroelastosis (Fig. 1d). On histology, convolutes composed of mature blood vessels were found embedded in fibrosis with focally inconspicuous inflammatory infiltrates and remnants of hypertrophied myocardium. Most vessels were large veins with prominent intimal hyperplasia and, to a lesser extent, tortuous malformed arteries, which rendered a diagnosis of AVM (Fig. 1e). Many veins showed thrombotic occlusion with different stages of organisation of thrombus. Large vessels were surrounded by numerous dilated thin-walled microvessels, but the immature type of capillary microvessels present in the initial myocardial biopsy specimen could be found only focally and after extensive tissue sampling. We interpreted this as maturation of the microvascular proliferative process. Vascular malformation was present only in the myocardial area fed by the right coronary artery.
Case No. 2

The second case is that of a 14-year-old boy, who had been known since birth to have asymmetrical hypertrophy of the ventricular septum, which was diagnosed at that time as hypertrophic cardiomyopathy (HCM). At the age of 11 years, cardioversion was performed for torsades de pointes, and he was treated with amiodarone to prevent further arrhythmias. At the age of 14 years, the patient was hospitalized for concomitant hyperthyroidism. During this hospital admission, the patient collapsed and died from untreatable ventricular fibrillation.

A formalin-fixed pathology specimen of the patient's heart (weight $900 \mathrm{~g}$ ) was sent to our hospital for consultation. Gross examination confirmed the presence of asymmetrical septal hypertrophy and protrusion of an anteroseptal mass, which had resulted in infundibular pulmonary stenosis (Fig. 2a). Sections taken at several levels from this mass showed convolutes of mature but malformed vessels, mainly large dilated veins and, to a lesser extent, tortuous arteries, which was consistent with a diagnosis of AVM. These vessels were embedded in an extensive solid mass of immature capillary vessels and focally embedded in collagen-rich fibrous tissue (Fig. 2b, c). These microvessels were morphologically and immunohistochemically similar to the microvascular proliferations observed in the myocardial biopsy specimen of patient no. 1. An interesting additional finding was the presence of distinct myocardial disarray in combination with fibrosis (Fig. 2d) within the area affected by AVM but 
Fig. 2 Patient no. 2. a Midventricular transverse section of the heart showing anteroseptal hypertrophy with congested hypervascular areas. b and c Histology (overview and details) showing large dilated and congested vessels surrounded by closely packed microvessels and sparse remaining cardiomyocytes (arrows). $\mathbf{d}$ and e Areas of myocardial disarray in the area affected by the vascular process and in adjacent nonaffected myocardium, respectively (b and $\mathbf{e}$ haematoxylin and eosin stain)
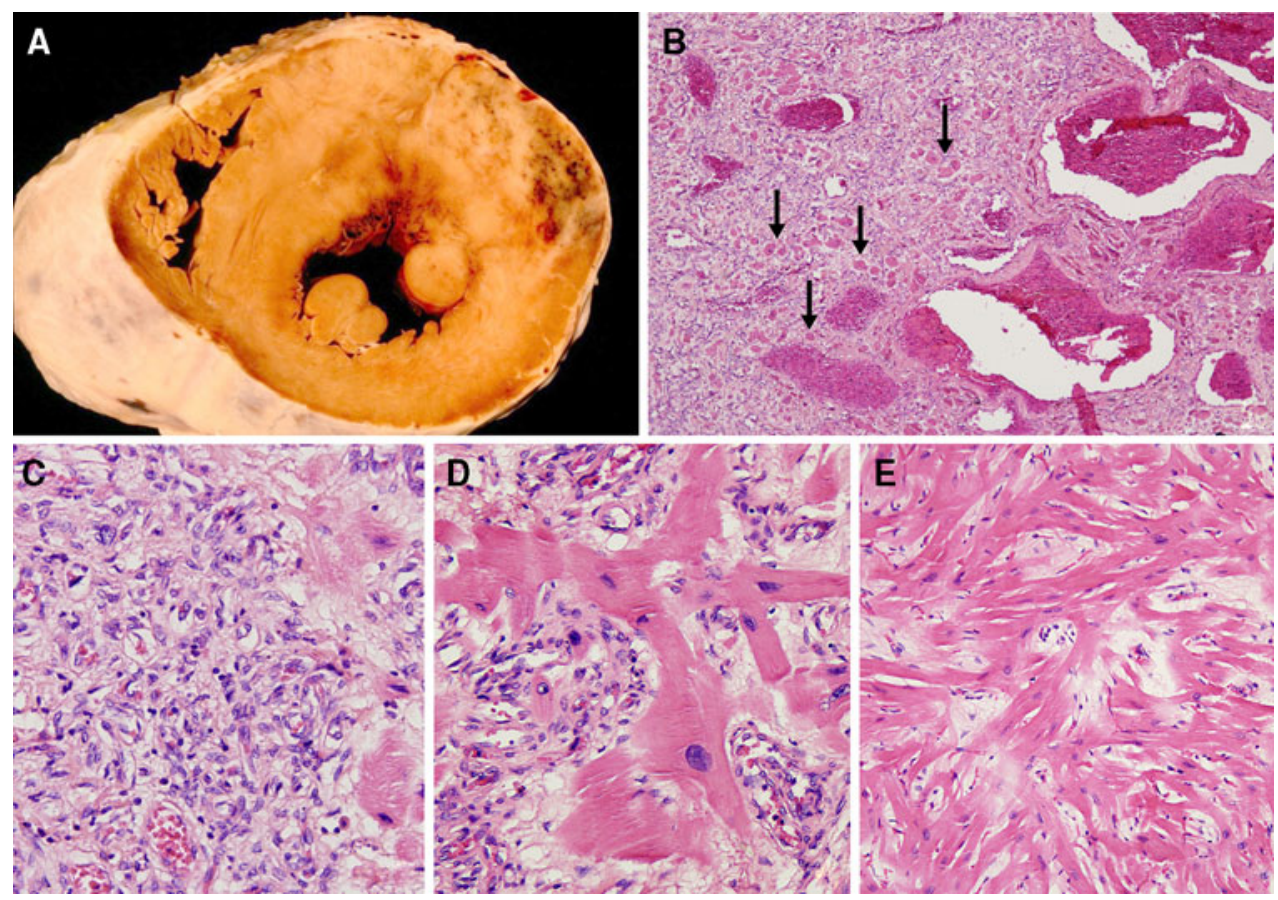

also in normal-appearing myocardium (Fig. 2e), which is in accordance with the initial clinical diagnosis at birth of HCM.

\section{Discussion}

Histopathological findings in the hearts of both patients are consistent with a diagnosis of intramural AVM [3, 7, 12]. Of particular interest in both cases, and not described earlier in hearts, is the additional component of immature capillary vessels within the boundaries of the congenital vascular lesion. Moreover, in patient no. 2, AVM coincided with histologically proven HCM. To our knowledge, such an association has not been previously reported.

In the literature, most cardiac vascular anomalies are termed "(hem)angiomas" and are classified according to the main type of vessel involved or their topographic localization within the heart (endocardial, intramuscular, or epicardial) [9, 10]. However, the general approach to classify all benign vascular cardiac tumours as (hem)angioma needs further specification. Currently, therefore, a more recent classification, as proposed by the International Society for the Study of Vascular Anomalies, is widely used in the diagnosis of vascular lesions of soft tissue and skin in children and adults. This classification endeavours to separate angiomas and congenital vascular malformations, which is desirable to enhance understanding of the clinical behaviour and prognosis of these lesions [5]. Vascular masses that are histologically composed of mature but malformed arteries and veins, as seen in our two patients, are in fact congenital vascular malformations (hamartomas) that show a slowly progressive type of growth and never regress [3,11]. Only a few cases of welldocumented cases of cardiac AVM composed of multiple dilated tortuous vessels have been described $[1,4,8]$. Of these, the case described by Schroeder et al. [8] is of particular interest because their description of AVM in a 7-year-old boy includes a similar network of immature capillary vessels amidst the large vessels of the malformation, as we noticed in our cases. Such benign microvascular proliferations associated with AVM have also been described in other anatomic locations, such as soft tissue and skin [6,7] and the gut [2]. In our study of 107 symptomatic vascular malformations of soft tissue and skin [7], we showed benign angioproliferative responses of varying intensity in $30 \%$ of patients with AVM but not in patients with other forms of vascular malformation, such as the purely lymphatic or venous types. This vasoproliferative effect has been interpreted as being due to local hypoxia in the microenvironment of the congenital malformation because tissue hypoxia is considered one of the strongest stimulating factors for angiogenesis. Albeit speculative, in the cases of AVM of the heart described here, tissue hypoxia generated in these increasingly hypertrophic and dilating hearts could also have played a role in the formation of angioproliferative masses.

Acknowledgments We thank Jan von der Thusen for editorial work with the manuscript.

Open Access This article is distributed under the terms of the Creative Commons Attribution Noncommercial License which 
permits any noncommercial use, distribution, and reproduction in any medium, provided the original author(s) and source are credited.

\section{References}

1. Aguilera B, Suárez-Mier MP, Argente T (2004) Cardiac arteriovenous malformation causing sudden death. Cardiovasc Pathol 13:296-298

2. Bavikatty NR et al (2001) Florid vascular proliferation of the colon related to intussusception and mucosal prolapse: potential diagnostic confusion with angiosarcoma. Mod Pathol 14:11141118

3. Calonje E, Weiss SW (2002) The WHO classification of tumours of soft tissue and bone, vol 5. International Agency for Research on Cancer Press, Lyon, France, pp 156-158

4. Kemme DJ, Rainer WG (1991) Subendocardial arteriovenous malformation in a patient with unstable angina. Clin Cardiol $14: 82-84$

5. Mackie AS, Kozakewich HP, Geva T, Perez-Atayde AR, Mulliken JB (2005) Vascular tumors of the heart in infants and children: case series and review of the literature. Pediatr Cardiol 26:344-349
6. Matsushige $\mathrm{T}$ et al (2004) Arteriovenous malformation of the scalp: case report and review of the literature. Surg Neurol 62:253-259

7. Meijer-Jorna LB, van der Loos CM, de Boer OJ, van der Horst CM, van der Wal AC (2007) Microvascular proliferation in congenital vascular malformations of skin and soft tissue. J Clin Pathol 60:798-803

8. Schroeder VA, Lee EY, Douglas WI, Cottrill CM (2007) Left coronary artery arteriovenous malformation presenting as a diastolic murmur with exercise intolerance in a child with a suspected familial vascular malformation syndrome. Congenit Heart Dis 2:203-207

9. Sobrinho AF, Ferreire JA, Borem MP, Oliveira JF, Borges LR, Dias MA et al (2005) Capillary-type cardiac hemangioma in the left atrium. Cardiovasc Surg 20:445-448

10. Sotoda Y, Hirooka S, Kohi M, Orita H, Mori M (2008) Intramuscular hemangioma in the right ventricle. Gen Thorac Cardiovasc Surg 56:85-87

11. Thomas JE, Eror AT, Kenney M, Carvalho Jr (2004) Asymptomatic right atrial cavernous hemangioma: A case report and review of the literature. J Cardiovasc Pathol 13:341-344

12. Vikkula M, Boon LM, Mulliken JB (2001) Molecular genetics of vascular malformations. Matrix Biol 20:327-335 